\title{
Intensification of mass transfer with chemical reaction under conditions of concentration- capillary convection
}

\author{
S.A. Ermakov ${ }^{1,2}$, A.A. Ermakov ${ }^{2, *}$, A.V. Mankov ${ }^{1}, Y u . R$. Muratov $^{1}$, and A.E. Koparulina ${ }^{1}$ \\ ${ }^{1}$ Ural State Agrarian University, Yekaterinburg, Russia \\ ${ }^{2}$ Ural Federal University named after the first President of Russia B.N. Yeltsin, Yekaterinburg, Russia
}

\begin{abstract}
The regularities of mass transfer with a chemical reaction in the system of aqueous solution of sodium hydroxide (solid medium) / butyric acid (transportable component) / carbon tetrachloride (dispersed phase) in spray extractors are studied. It is shown that the resulting chemocapillary instability can significantly reduce the height of the transfer unit. The conditions for the occurrence of spontaneous concentration-capillary convection during mass transfer with a chemical reaction under conditions of constrained drop movement are determined.
\end{abstract}

\section{Introduction}

One of the ways to increase the efficiency of gravity extractors is to conduct mass transfer processes in the interfacial instability mode. In relation to the operation of gravity extractors, the mass transfer process under conditions of spontaneous interphase convection (SIC) will make it possible to significantly intensify the extraction processes, which will reduce the transfer unit height (TUH), i.e., the height of the column apparatus. Experiments conducted to study mass transfer with chemical reaction (in the absence of a chemical reaction) at the spherical prase boundary [1] showed that the mass transfer rate significantly depends on the SIC that occurs in the extraction system. As shown by the conducted studies, the mass transfer coefficient in the conditions of concentration-capillary convection can increase by 2-10 times [1].

In gravity extractors, when one of the phases is dispersed, the effect of concentrationcapillary convection on the kinetics of mass transfer with chemical reaction is likely to be different than when transferring from a single drop (to a drop). These patterns are associated with the fragmentation and fusion of drops, and as a result of this change in the interfacial surface, their size in height and cross-section of the apparatus. As it is known, longitudinal mixing can have a significant impact on the driving force of the mass transfer process, which is due to the uneven distribution of flows across the cross-section of the apparatus, based on this, longitudinal mixing can affect the intensity of mass transfer in interphase instability mode and SIC driving force. This is confirmed by the experiments

\footnotetext{
* Corresponding author: piap.kafedra@mail.ru
} 
conducted, which showed that the mass transfer coefficient under SIC conditions is a function of the driving force [2,3].

\section{Purposes and objectives}

Thus, it is necessary to determine whether there is a concentration-capillary instability during mass transfer with chemical reaction under conditions of constrained drop movement in gravity extractors? How does the concentration-capillary instability affect the intensity of mass transfer with a chemical reaction and the efficiency of column extractors?

\section{Materials and methods}

The experiments were carried out on an extraction system: the giving phase is carbon tetrachloride, the receiving phase is aqueous solution of sodium hydroxide, the transferred substance is butyric acid. In this system, mass transfer with chemical reaction in the interfacial instability mode is studied in detail on a single drop [1].

The studies were carried out in a gravity-controlled spray-type extractor (Fig. 1), $d_{\text {ap. }}=$ $3.1 \cdot 10^{-2} \mathrm{~m}$, height $\mathrm{h}_{\text {ap. }}=0.6 \mathrm{~m}$, working volume $\mathrm{V}_{\text {ap. }}=4.41 \cdot 10^{-4} \mathrm{~m}^{3}$ at a temperature $\mathrm{t}=$ $20 \pm 0.1{ }^{\circ} \mathrm{C}$. The movable feeding tube had a distributor nozzle with 16 holes with a diameter of $\mathrm{d}_{\text {hol. }}=1.0 \cdot 10^{-3} \mathrm{~m}$. The drop collector for collecting the dispersed phase had a diameter $\mathrm{d}_{\mathrm{col}}=7.0 \cdot 10^{-3} \mathrm{~m}$. The volume of the dispersed phase was $\mathrm{V}_{\mathrm{dp}}=500 \mathrm{ml}$, the solid phase was $\mathrm{V}_{\mathrm{dp}}=2000 \mathrm{ml}$, and the sample volume was $\mathrm{V}_{\mathrm{df}}=2 \mathrm{ml}$.

In the course of scientific research, reagents of the H.C. and C.D.A. brands were used. The purity of the reagents was controlled by the boiling point and refractive index.

To exclude the influence of mass transfer of solvents on the kinetic regularities of mass transfer of the main substance, the organic and aqueous phases were mutually saturated before the experiment (within 12 hours). The binder was dissolved in the aqueous phase, and the transferred substance was dissolved in the giving (organic) phase.

The experiment was carried out in the following sequence: a capillary with a distributor nozzle (3) was set to a certain height using a movable feeding tube (4). The dispersed phase was supplied by gravity from a Marriotte vessel (5). After passing a given height, the drops were collected by a drop catcher (6), from where the phase was continuously withdrawn, while the level of the interfacial surface mirror remained constant. When the column reached the stationary mode of operation, samples were taken and analyzed. The aqueous solution of sodium hydroxide was periodically replaced with a fresh solution with a constant concentration [1]. In order to eliminate the influence of longitudinal mixing during dispersion, the solid phase was kept in a motionless state. To obtain the kinetic dependence "the concentration of the transferred substance - the column height", the capillary with the nozzle-distributor was moved to a new point-height. The experiment was carried out leaving unchanged the hydrodynamic and physico-chemical parameters of the contacting phases. In order to ensure a dispersion rate favorable for the formation of equal-sized drops, the flow rate of the dispersed phase was selected in a certain way [1].

The speed of the "free" movement of the drops was determined by the method described in $[4,5]$, using a high-speed video camera with a shooting frequency of $50 \mathrm{~Hz}$. An image processing tool (Image-Pro Plus 6.0) was used to analyze the recorded sequence of frames.

The retention capacity of the extraction column (the volume of the dispersed phase in drops in the working volume of the apparatus) was determined by the method [6].

The concentration of the binder reagent in the solid phase volume was considered constant, since the proportion of the dispersed phase passing through the column at one measurement was less than $5 \%$ of the solid phase volume. 


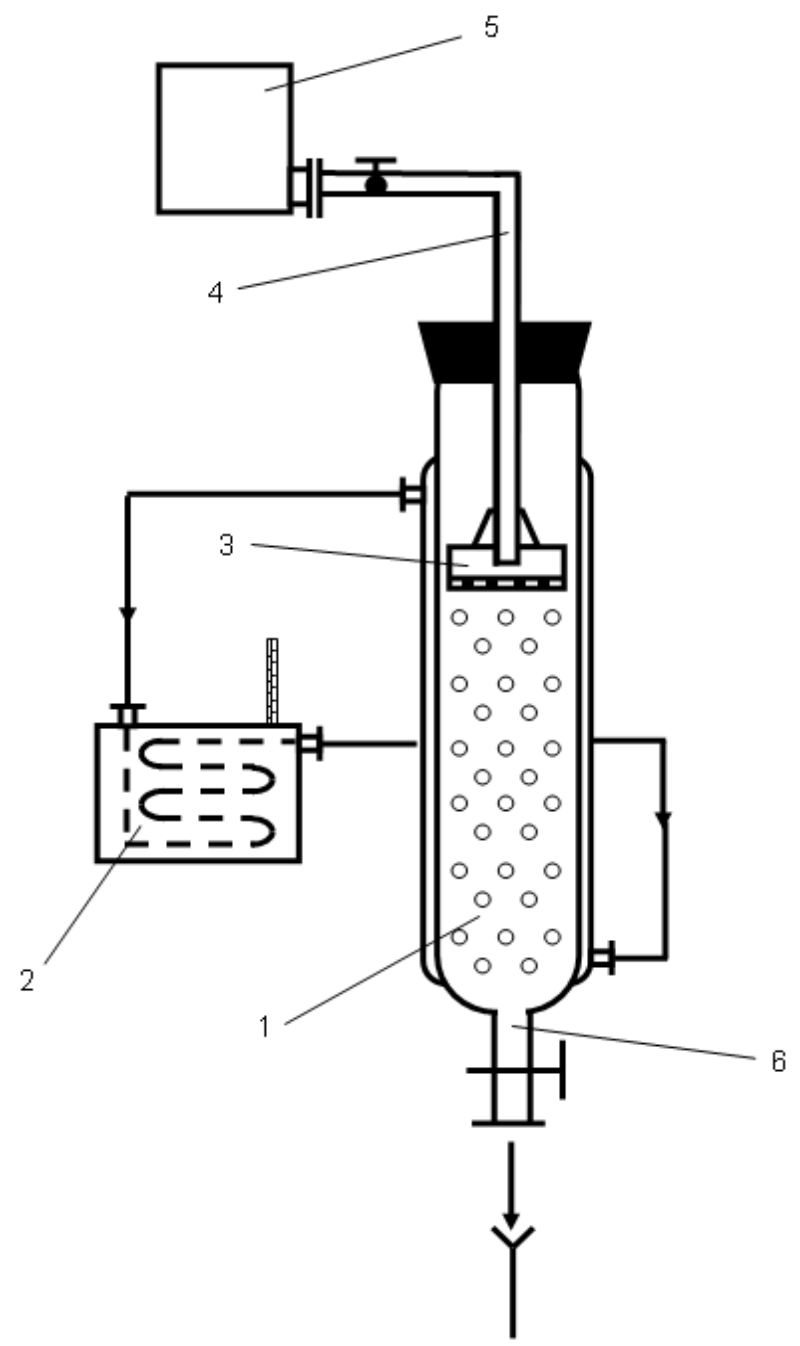

Fig. 1. Laboratory installation for studying the kinetics of mass transfer in a spray column: 1 column; 2 - thermostat; 3 - nozzle-distributor; 4 - feeding tube; 5 - Marriotte vessel; 6 - sampler.

Processing of experimental data.

To process the experimental data, graphical dependences were constructed in the coordinates of the transferred substance concentration $\mathrm{c}-$ the height of the column $\mathrm{h}$. The "free" movement time of the drops, otherwise the process time $(\tau)$, was calculated by knowing the distance that the drops traveled from the distributor nozzle to the interfacial surface mirror and the "free" movement speed of the drops in the continuous phase.

The experimental data were approximated by a two-exponential function using the least square method, in the coordinates the transferred substance concentration $\mathrm{c}-$ the process time $\tau$.

The averaged values of the process speed and mass transfer coefficients were found using the obtained kinetic curve according to the equation:

$$
-\frac{d c}{d \tau}=K_{M} \cdot S_{s p} \cdot c
$$


where $\mathrm{K}_{\mathrm{M}}$ is the mass transfer coefficient, $\mathrm{m} / \mathrm{s} ; \mathrm{S}_{s p \text {. }}$ - the specific contact surface of the phases, $\mathrm{m}^{2} / \mathrm{m}^{3}$; c - the driving force of mass transfer, equal to the transferred substance concentration in the volume of the limiting (giving) phase [7], $\mathrm{kmol}^{3} \mathrm{~m}^{3} ; \tau$ - the process time, $s$.

The specific contact surface of the phases in the spray column was determined by the formula:

$$
S_{s p}=\frac{V_{d r} \cdot 6}{V_{a p} \cdot d_{s v}}
$$

where $\mathrm{V}_{\mathrm{dr}}$ is the average volume of the dispersed phase in drops, $m^{3} ; \mathrm{V}_{\text {ap }}$ is the working volume of the column, $m^{3} ; \mathrm{d}_{\mathrm{sv}}$ is the surface-volume diameter, $m$.

The average drop surface-volume diameter was calculated:

$$
d_{S V}=\frac{\sum d_{\text {drop }}^{3}}{\sum d_{\text {drop }}^{2}}
$$

where $\mathrm{d}_{\text {drop }}$ is the drop diameter, $m$.

The average drop diameter was determined from the known volume of the dispersed phase and the number of drops formed from this volume of the dispersed phase.

The SIC intensity and the conditions for the interphase instability occurrence were judged by the intensity coefficient $\left(\mathrm{K}_{S I C}=\operatorname{tg} \alpha\right.$, where $\alpha$ is the angle of inclination of the straight line in the coordinates of the mass transfer coefficient - the driving force of the process) and the critical concentration $\left(\mathrm{c}_{\mathrm{cr}}\right)$ - the parameters of the empirical equation describing the mass transfer process under SIC conditions (Fig. 3) [8]:

$$
J=\left\{\begin{array}{c}
{\left[K_{D}+K_{S I C} \cdot\left(\mathrm{c}-\mathrm{c}_{c r}\right)\right] \cdot c, \text { at } c>c_{c r}} \\
K_{D} \cdot c, \text { at } c \leq c_{c r},
\end{array}\right.
$$

Where $\mathrm{D}$ is the coefficient of mass transfer in the "diffusive" regime (diffusionconvection mechanism), $\mathrm{m} / \mathrm{s} ; \mathrm{K}_{\text {SIC }}$ - coefficient taking into account the acceleration of mass transfer in interfacial instability mode, $\mathrm{m}^{4} / \mathrm{kmol} \cdot \mathrm{s} ; \mathrm{c}$ - the driving force of the mass transfer process, $\mathrm{kmol} / \mathrm{m}^{3} ; \mathrm{c}_{\mathrm{cr}}-$ critical driving force (critical concentration), $\mathrm{kmol} / \mathrm{m}^{3}$. Equation parameter $\left[\mathrm{K}_{\mathrm{D}}+\mathrm{K}_{S I C} \cdot\left(\mathrm{c}-\mathrm{c}_{\mathrm{cr}}\right)\right]=\mathrm{K}_{\mathrm{M}}-$ mass transfer coefficient in the interfacial instability mode, $m / s$.

To evaluate the efficiency of the extraction column, the value TUH - the height of the transfer unit, was used:

$$
T U H=\frac{V_{d f .}}{K_{M} \cdot S_{s p} \cdot f},
$$

where $\mathrm{V}_{\text {d.f. }}$ is the flow rate of the dispersed phase, $\mathrm{m}^{3} / \mathrm{s} ; \mathrm{K}_{\mathrm{M}}$ is the mass transfer coefficient, $\mathrm{m} / \mathrm{s} ; \mathrm{S}_{s p}$. is the specific contact surface of the phases, $\mathrm{m}^{2} / \mathrm{m}^{3} ; \mathrm{f}$ is the crosssectional area of the apparatus, $m^{2}$.

It can be assumed that in the presence of concentration-capillary instability in the system, the height of the transport unit consists of two values: $\mathrm{TUH}_{\text {total }}=\mathrm{TUH}_{\mathrm{SIC}}+\mathrm{TUH}_{\mathrm{diff}}$, where $\mathrm{TUH}_{\text {sic }}$ - corresponds to interfacial instability mode; $\mathrm{TUH}_{\text {diff }}$ - to the "diffusion" 
mode. The average value of the mass transfer coefficient, at which the value of the TUH $\mathrm{TIC}_{\mathrm{S}}$ was determined, was calculated:

$$
\mathrm{K}_{a v}=\frac{K_{\mathrm{M}}^{0}+K_{D}}{2}
$$

The "initial" values of the mass transfer coefficient $\left(\mathrm{K}_{\mathrm{m}}^{0}\right)$ were estimated by extrapolating the function (7) to the zero point in time on the graphical dependence of the mass transfer coefficient - the process driving force [9].

$$
K_{M}=-\frac{d c(\tau)}{d \tau} \cdot \frac{1}{S_{s p} \cdot c(\tau)}
$$

Calculation of the $\mathrm{TUH}_{\text {diff. was }}$ carried out at the mass transfer coefficient for the "diffusion" mode $\left(\mathrm{K}_{\mathrm{D}}\right)$.

During scientific research, the following assumptions are made: the shape of the drops is spherical, the surface of the drops is not deformed, the size and shape of the drops does not change during the process.

\section{Research results}

As can be seen from the above graphs (Fig. 2), with an increase in the initial concentration of the transferred substance $\left(\mathrm{c}_{0}\right)$, the intensity of the concentration-capillary instability $\left(\mathrm{K}_{S I C}\right)$ decreases, and the critical concentration $\left(\mathrm{c}_{\mathrm{cr}}\right)$ increases. The increase in the initial concentration of the transferred substance leads to a decrease in the interfacial tension of the system, and as a consequence, a decrease in the gradients of the interfacial tension and the SIC intensity, which is in good agreement with the data obtained earlier on the spherical and flat interfacial boundaries [2].

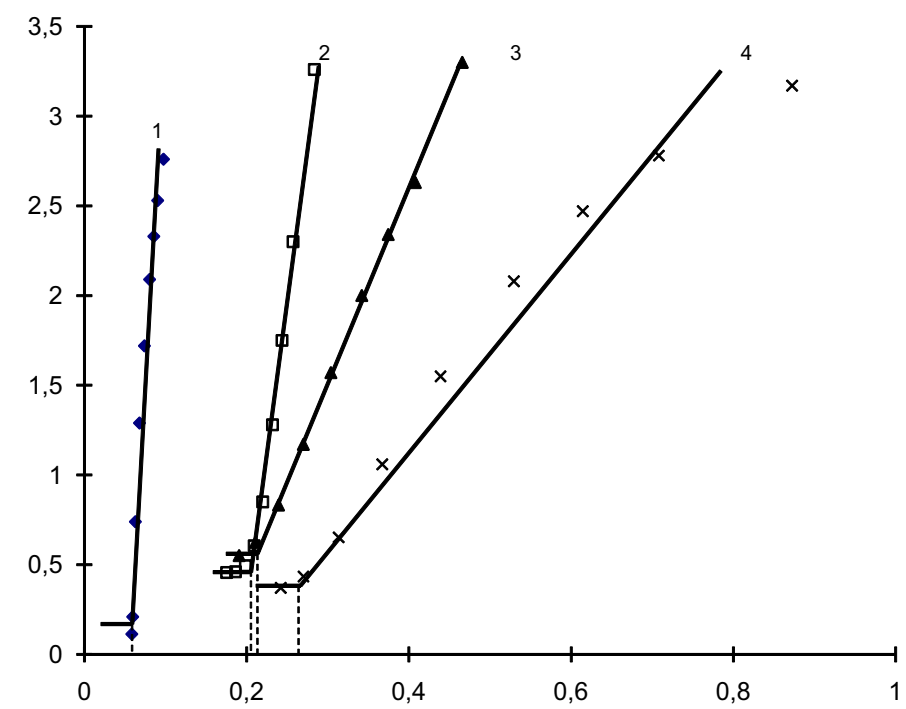

Fig. 4. The dependence of the intensity (Ksic) and the conditions for the occurrence (Ccr) of spontaneous interphase convection in the spray column on the initial concentration of the transferred substance. 
Mass transfer of butyric acid from carbon tetrachloride to an aqueous $\mathrm{NaOH}$ solution $(\mathrm{CNaOH}=2.0 \mathrm{kmol} / \mathrm{m} 3)$ :

$1-\mathrm{C} 0=0.099 \mathrm{kmol} / \mathrm{m} 3$;

$2-\mathrm{C} 0=0.302 \mathrm{kmol} / \mathrm{m} 3$;

$3-\mathrm{C} 0=0.501 \mathrm{kmol} / \mathrm{m} 3$;

$4-\mathrm{C} 0=0.990 \mathrm{kmol} / \mathrm{m} 3$.

Table 1 shows the initial concentration effect of the transferred substance on TUH value in the spray column. The data analysis in Table 1 shows that an increase in the initial concentration of the transferred substance $\left(\mathrm{c}_{0}\right)$ reduces the value of $\mathrm{TUH}_{\text {total }}$. The obtained regularities are explained by an increase in the amount of substance that is transferred in interphase instability mode. As can be seen from the data in Table 1, the share of mass transfer under SIC conditions accounts for from $30 \%$ to $70 \%$ of the amount of transferred substance, which contributes to a significant reduction in the value of the $\mathrm{TUH}_{\text {total }}$.

Table 1. The dependence of the TUH value on the initial concentration of the transferred substance in the spray column (system carbon tetrachloride + butyric acid - aqueous $\mathrm{NaOH}$ solution $\left(\mathrm{c}_{\mathrm{b} . \mathrm{r} .}=2.0\right.$

$\left.\left.\mathrm{kmol} / \mathrm{m}^{3}\right)\right)$

\begin{tabular}{|c|l|l|l|l|l|l|l|}
\hline $\begin{array}{l}\mathrm{c}_{0}, \\
\mathrm{kmol} / \\
\mathrm{m}^{3}\end{array}$ & $\begin{array}{l}\text { Amount of } \\
\text { substance } \\
\text { transferred } \\
\text { in the } \\
\text { interfacial } \\
\text { instability } \\
\text { mode, } \%\end{array}$ & $\begin{array}{l}\text { Amount } \\
\text { of } \\
\text { substance } \\
\text { transferre } \\
\mathrm{d} \text { in the } \\
\text { "diffusion } \\
\text { mode, } \\
\%\end{array}$ & $\begin{array}{l}\text { Dispersio } \\
\text { n phase } \\
\text { flow rate, } \\
\mathrm{V}_{\mathrm{d}} \cdot 10^{5}, \\
\mathrm{~m}^{3} / \mathrm{s}\end{array}$ & $\begin{array}{l}\text { Specifi } \\
\text { c phase } \\
\text { contact } \\
\text { surface, } \\
\mathrm{m}^{2} / \mathrm{m}^{3}\end{array}$ & $\begin{array}{l}\mathrm{TUH}_{\mathrm{SIC}} \\
\left(\mathrm{at} \mathrm{k}_{\mathrm{m}}=\right. \\
\left.\mathrm{k}_{\mathrm{av}}\right), \\
\mathrm{m}\end{array}$ & $\begin{array}{l}\mathrm{TUH}_{\text {diff. }} \\
\left(\mathrm{at} \mathrm{k}_{\mathrm{m}}=\mathrm{k}_{\mathrm{D}}\right), \\
\mathrm{m}\end{array}$ & $\begin{array}{l}\mathrm{TUH}_{\text {total }} \\
\mathrm{m}\end{array}$ \\
\hline 0.099 & 49.49 & 50.51 & 0.188 & 16.0 & 1.08 & 13.62 & 7.41 \\
\hline 0.302 & 33.86 & 66.14 & 0.188 & 20.0 & 0.67 & 2.73 & 2.03 \\
\hline 0.501 & 58.08 & 41.92 & 0.188 & 23.6 & 0.55 & 1.92 & 1.12 \\
\hline
\end{tabular}

\section{Conclusions}

Thus, the existence of concentration-capillary instability under conditions of constrained drop movement was proved. It is shown that the resulting interfacial instability intensifies the substance transfer process, increasing the mass transfer coefficient by 2-10 times.

Experiments have shown that in column extractors, the intensity of concentrationcapillary convection decreases with an increase in the initial concentration of the transferred substance, and the critical concentration increases.

The efficiency of the extraction columns increases when concentration-capillary instability occurs in the system, that is, the height of such apparatuses decreases.

\section{References}

1. S.A. Ermakov, Mass transfer with chemical reaction under conditions of spontaneous interphase convection in liquid extraction processes, Abstract. of doctor. th. Yekaterinburg, 40 (2006)

2. S.A. Ermakov, A.A. Ermakov, V.A. Stepanov, ZhPH, 77(11), 1847-1852 (2004)

3. R. Treybal, Liquid extraction, 724 (Moscow, Chemistry, 1966)

4. M. Wegener, M. Kraume, A.R. Paschedag, AIChE Journal, 56 (1), 2-10 (2010) 
5. E. Bertakis, S. Gross, J. Grande et al. Chem. Eng. Science, 65, 2037-2051 (2001)

6. A.A. Ermakov, Intensification of mass transfer under conditions of spontaneous interphase convection during liquid extraction. Abstract. of doctor. th. Moscow, 48 (2015).

7. J. Astarita, Mass transfer with chemical reaction, 223 (L.: Chemistry, 1971)

8. A.A. Ermakov, Yu.A. Konshin, V.I. Nazarov, ZhFH, 51(8), 2151 (1977)

9. T.K. Sherwood, J.C. Wei, Industrial and Engineering Chemistry, 49, 1030-1034 (1957) 